\title{
PHOTOELECTRIC REMOTE DETECTION TECHNOLOGY IN ROBOT MANUFACTURING
}

\author{
Xiangyun $\mathrm{Jia}^{1}$ \\ ${ }^{1}$ Rizhao Polytechnic, Mechanical and Electrical Engineering Institute, Rizhao, Shandong, 276826, China. \\ Email: jiao646@sina.com
}

\begin{abstract}
In order to understand that the intelligent robot has certain space exploration ability, in this study, the fiber-optic sensors in robot manufacturing are analyzed, and then the remote detection technology is studied. The research results show that when the photoelectric remote detection technology is applied to the robot manufacturing, the optical fiber sensor can provide more detailed data information for the robot, and enable the robot to understand the detection situation in time, so as to provide effective basis for the rear managers and decision makers. Therefore, it is of great significance for the innovation and development of intelligent robot to study the photoelectric remote detection technology in robot manufacturing.
\end{abstract}

Keywords: Photoelectric Remote Detection; Intelligent Manufacturing; Robot.

\section{Introduction}

At the beginning of the 21st century, China's manufacturing industry is facing the bottleneck of the disappearance of demographic dividend, rising labor price and overcapacity. With the continuous development of industry, under the background of industry 4.0, intelligent manufacturing has become a new direction of the development of China's manufacturing industry, and robot has become the main content of intelligent manufacturing. Therefore, in order to promote the transformation and upgrading of China's manufacturing structure, China has formulated the "made in China 2025" strategy to enhance and maintain the competitiveness of China's manufacturing in the world market. Vigorously developing robot industry is the main direction of the strategy. Nowadays, intelligent robot technology has been widely used in many fields, such as production, life and so on. Especially in the 21st century, with the rapid development of information technology, intelligent control technology and other technologies, the research of robot technology has become an important content in many fields [1].

At present, the Chinese robot market is facing great challenges and opportunities. On the one hand, there is still a big gap between the research and development capability of Chinese robots and that of foreign ones, and the international market will face more fierce competition in the future. On the other hand, China's robot market is also facing greater opportunities. With the continuous growth of the national economy, it is necessary to constantly adjust the industrial structure, actively change the development mode, let more intelligent products into people's lives and various fields, and help people solve problems and difficulties. At the same time, the strong support of national policies, the disappearance of population and labor dividends, the sharp increase of market demand, the industry user awareness and the diversification of industry applications all create an unprecedented good environment and opportunity for the rapid development of China's robot industry. The application of robot has been greatly improved from the field to the performance level. It is also a high demand for the continuous development of robot industry to broaden the application field and improve the performance level. Only by complementing each other and promoting each other, can the performance and intelligence of the robot be greatly improved. At the same time, the application field of the robot can be continuously expanded, so that the robot can help human to complete more tasks that are difficult to complete before.

Photoelectric detection technology is a kind of technology to detect and recognize the object according to the characteristics of the light wave radiated or reflected by the detected object. This technology itself can play its advantages of clearer, more accurate, faster response and stronger survivability in military applications. At the same time, photoelectric detection technology also includes photoelectric reconnaissance, night vision, navigation, guidance, homing, search, tracking and identification functions. In addition, with the continuous development of China's economy, various fields have developed rapidly. Especially in 
the field of aeronautics and astronautics, rapid space platforms such as reconnaissance and exploration satellites, high-altitude and long-term aircraft, supersonic missiles and so on have increasingly strong demand for long-range detection of photoelectric loads, which not only can provide more front-line intelligence for this field, but also can help relevant departments to make timely decisions in the face of emergency [2-3].

Therefore, in this study, considering the wide application of intelligent robots in the future, photoelectric remote detection technology can give robots more functions. In this study, the photoelectric remote detection technology is studied in robot manufacturing, in order to have certain significance for the future development of robot manufacturing and the development of photoelectric remote detection technology.

\section{Literature Review}

The manufacturing of intelligent robot brings more possibilities for the industrial manufacturing of our country and provides technical support for the development of intelligent life.

$\mathrm{Hu}$ et al. (2019) proposed a new robot factory system architecture from six aspects in the research, including intelligent terminal, system management, edge computing node, cloud cognition engine, intelligent device unit and robot factory production line layer. Through the discussion of effective information interaction, multi-mode data fusion and automatic production in machine manufacturing, the solution of interactive modeling and active operation and maintenance based on edge computing and communication is proposed. The research results show that the robot factory has significantly improved chip assembly and production efficiency, and the number of system instructions has also been greatly reduced [4]. Huang et al. (2018) proposed a new robot scheme with dynamic high-speed compensation system, and compared it with the traditional multi joint industrial robot. The research results show that the traditional multi joint industrial robot is designated for fast and rough global motion, and the direct driven add-on module is realizing the local compensation for the accumulated uncertainty. Moreover, the new highspeed vision system can realize image sensing and processing of $1000 \mathrm{fps}$, and the total delay time of high-speed vision feedback is within 3.0ms [5].

$\mathrm{Hu}$ et al. (2018) aimed to obtain a pin silicon photodetector array with high quantum efficiency, analyzed the important factors affecting the quantum efficiency and dark current of the pin silicon photodetector array, and carried out theoretical analysis and simulation research on the antireflection layer structure of the incident surface of the detector. The results show that the dark current of single pixel of LPD silicon photodetector array is lower than that of Binsong S11212 commercial photodetector [6]. Pan et al. (2019) studied that in order to improve the charge separation and transfer of effective excitons in perovskite nanocrystals, perovskite nanocrystals were grown in situ on two-dimensional mxene nanocrystals, and then new functional nanocomposites were synthesized. When photodetectors or photocatalysts are used to detect the structure of materials, the generation of photocurrent is increased, which also proves the potential application of these heterostructure nanocomposites in photoelectric detection [7].

To sum up, the previous research is mainly from the aspects of robot manufacturing, robot intelligent system, materials, aerospace and military operations. Photoelectric remote detection technology has not been applied to robot manufacturing. Therefore, in this study, the photoelectric remote detection technology in robot manufacturing is studied.

\section{Methods and Experiments 3.1 Freedom of main structure}

In robot manufacturing, robot kinematics studies the motion characteristics of the robot, regardless of the force exerted when the robot generates motion. It is necessary to study the position, velocity, acceleration, position and flexibility of the main components of the robot. The response frequency and motion speed of the robot will affect the working efficiency of the robot, which is also a very important content in the later application. In order to improve the flexibility of each part of the robot, the degree of freedom is mainly considered. The degree of freedom of mechanism refers to the number of independent movements of mechanism. Generally, when the active part of the mechanism is equal to the number of degrees of freedom, the mechanism has a certain relative motion. In the mechanical principle, the plane degree of freedom can be expressed by equation (1):

$$
F=3 n-3 P_{L}-P_{H}
$$

In the equation, $\mathrm{n}$ represents the number of members of the planar mechanism, and $\mathrm{P}_{\mathrm{L}}$ represents that the contact point of the planar mechanism is a line or a plane motion pair, that is, the motion low pair. PH represents point contact kinematic pair of planar mechanism, i.e. kinematic high pair [8]. There are many parallel mechanisms in robot manufacturing, but it is relatively difficult to calculate the degree of freedom. Therefore, the modified equation (2) of spiral theory is used to calculate:

$$
M=d(n-g-l)+\sum_{i=1}^{g} f_{i}+v
$$


In the equation, $\mathrm{M}$ is the number of degrees of freedom of the mechanism, $d$ is the order of the mechanism, and $d=6-\lambda . n$ represents the total number of components (including rack). $\mathrm{g}$ is the total number of pairs of motion. fi is the degree of freedom of the $\mathrm{i}$-th kinematic pair. $\mathrm{v}$ is the redundant constraint of parallel mechanism [9].

\subsection{Photodetector}

Photodetector is the core part of the detection system. According to the wavelength and intensity of the optical signal reaching the detector, the parameter requirements of the photodetector in the spectrum diagnosis system are different. It is very important to understand the working principle and characteristics of different photodetectors for the design of the detection system. The working principle and characteristics of commonly used photodetectors are briefly analyzed and compared. Commonly used photodetectors include photodiode (PPD), avalanche photodiode (APD), photomultiplier tube (PMT), charge coupled detector (CCD), C-MOS detector, etc. The detection range of photodetector is mainly determined by its noise characteristics for a specific wavelength of optical signal. The output signal of the detector should be at least larger than its intrinsic noise. Therefore, it is necessary to realize the noise characteristics of various detectors. In the semiconductor model of photodiode, P-type semiconductor and N-type semiconductor form PN junction. In the semiconductor, the direction of the internal electric field is from the $\mathrm{N}$ region to the $\mathrm{P}$ region, resulting in a small number of carriers moving along the electric field direction, opposite to the diffusion direction [10]. When light hits a semiconductor material, if the photon energy is larger than the gap of the semiconductor material, the photon energy is absorbed by the valence electron in the semiconductor atom, and the electron is excited into the conduction state, then the electron hole pair is generated. Not all electron hole pairs can form an electric current. The electron hole pair in the depletion region of the PN junction is the main part of the photocurrent, which accelerates the electron.

The semiconductor model is shown in Figure 1:

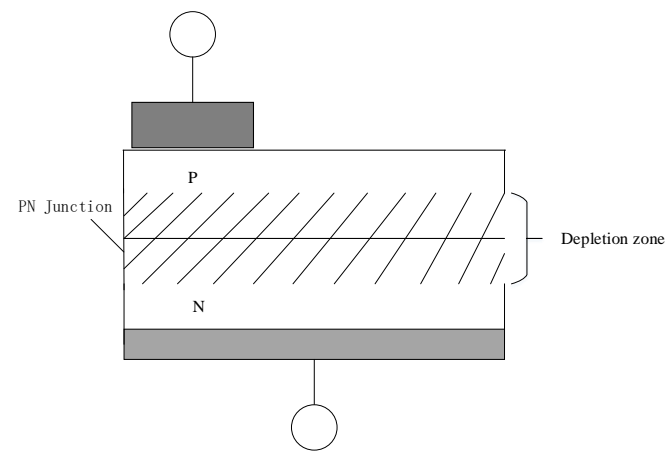

Figure 1: Semiconductor model of photodiode
In the equivalent analog circuit in the diode, the output current is as shown in equation (3):

$$
\begin{aligned}
I_{O} & =I_{1}-I_{D}-I^{\prime} \\
& =I_{L}-I_{S}\left(\exp \left(\frac{q V_{D}}{k T}\right)-1\right)-I
\end{aligned}
$$

In the equation, $\mathrm{I}_{\mathrm{L}}$ is the photo generated current caused by incident light, $V_{D}$ is the voltage at both ends, $I_{D}$ is the dark current flowing through photodiode, $C_{j}$ is the junction capacitance of PN junction, $R_{s h}$ is the equivalent series resistance of photodiode, $V_{0}$ and $I_{0}$ are the output voltage and output current, IS is the reverse saturation current of photodiode, $\mathrm{q}$ is the electronic charge, $\mathrm{k}$ is the Planck constant, and $\mathrm{T}$ is the photodiode temperature.

The detection range of photodiode is mainly affected by the wavelength of optical signal and its intrinsic noise. With different wavelengths, the quantum efficiency of photodiodes is obviously different. There are three kinds of total noise.

The first is thermal noise of the equivalent resistance Rsh, the second is shot noise caused by dark current, and the third is shot noise caused by photocurrent.

According to different types of detection, photodetectors can be divided into image detection and point detection. Among them, image detection is the use of photosensitive elements to sense the spatial change of light intensity information, and then imaging to produce intuitive image information.

Point detection generally refers to the conversion of optical signals into electrical signals for processing or acquisition [11].

In image detector, charge coupled device (CCD) is the most commonly used two types of image detector. In this study, CCD image is used for imaging. CCD is a shift register made up of a metal oxide semiconductor capacitor array arranged according to certain rules.

Using the photoelectric and charge transfer functions of CCD, a CCD image sensor is made.

The sensor is divided into line array and face array.

The structure of the area array image sensor is shown in Figure 2:

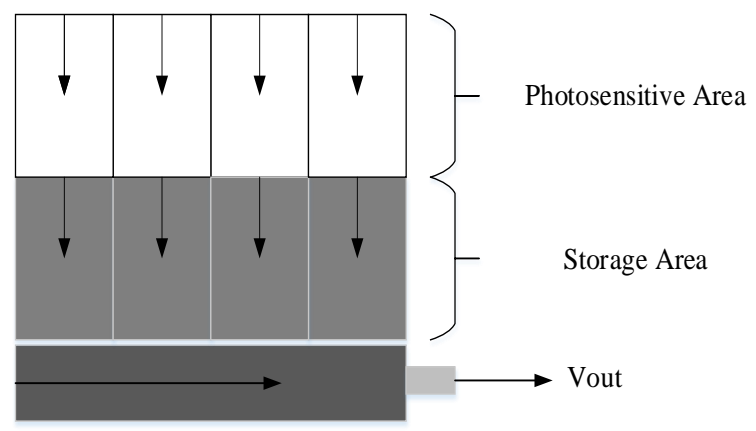

Figure 2: CCD image sensor 
In the above figure, figure a shows the frame transmission mode, and figure $b$ shows the inter line transmission array. In figure $b$ mode, the charge transfer distance is short, the chip size is small, the working frequency is high, and the unit structure is complex. If the back is illuminated, it will interfere and will not work.

\subsection{Analysis of photoelectric remote detection technology in robot manufacturing}

In the analysis of this technology, the main parameters to be considered are sensitivity, resolution, transfer efficiency, operating frequency and dark current characteristics. Sensitivity is the primary characteristic of the sensor, which can sense the external light intensity, marking the photoelectric conversion efficiency of the photosensitive region during the period, and finally expressed by the current or voltage amplitude of the output signal of the device. Resolution is the ability of the image sensor to distinguish the light and dark details in the image. It is usually expressed by limiting resolution or modulation transfer function (MTF). Considering the actual situation, MTF is used to express in this study. This function generally represents the imaging clarity of the optical imaging system, as shown in equation (4):

$$
\operatorname{MTF}(v)=M_{\text {out }}(v) / M_{\text {in }}(v)
$$

Among them, $\mathrm{M}_{\text {out }}(\mathrm{V})$ represents the modulation of the output signal, and $M_{\text {in }}(V)$ represents the modulation of the input optical signal. The regulation M can be expressed as equation (5):

$$
M=\frac{A_{\max }+A_{\min }}{A_{\max }-A_{\min }}
$$

In the equation, $A_{\max }$ represents the maximum value of the modulated wave signal and $A_{\text {min }}$ represents the minimum value of the modulated wave signal. When $\mathrm{v}=0$, the device has no loss in the transmission process. When MTF takes the maximum value of 1 , with the increase of spatial frequency, MTF value decreases gradually. Because of the system, when the MTF value is reduced to a certain value, the image cannot be clearly distinguished. The spatial frequency corresponding to this value is the highest spatial frequency that the image sensor can distinguish [12]. In general, when the MTF value drops to $10 \%$ of the corresponding logarithm of the line, that is the limit resolution of the positioning image sensor.

The CCD imaging system integrates the signal processor and the CCD image detector on the same substrate, which constitutes the core of the imaging system. When the photosensitive unit receives the image input, it will produce different strong and weak charges. Driven by the transfer voltage, the high-speed output of the charge is transmitted to the television receiver according to certain rules, and then the static optical image formed in each instant is displayed.

The principle of photoelectric remote detection is shown in Figure 3:
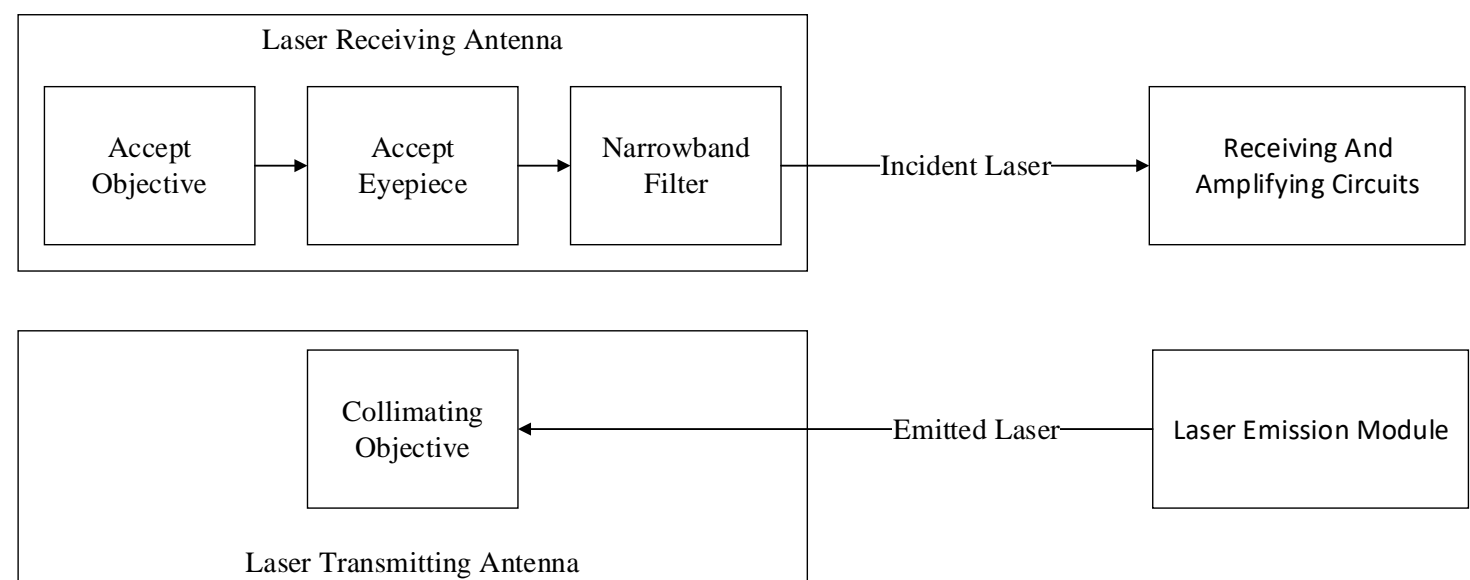

Figure 3: Principle of photoelectric remote detection

In addition, point detector is needed to realize photoelectric signal conversion. This is because in the process of remote detection, distance measurement is also very important. Avalanche photodiode (APD) is an important tool in point detector used in laser ranging system. It is a kind of high-speed photoelectric device that produces avalanche multiplication effect under the action of strong electric field at both ends. In laser ranging system, spectral responsivity, rise time and multiplication factor should also be considered. Spectral responsivity refers to the photoelectric conversion response of APD to various wavelengths of laser. According to the wavelength determined by the laser ranging system, the spectral responsivity of the detector is selected. 
The image is shown in Figure 4:

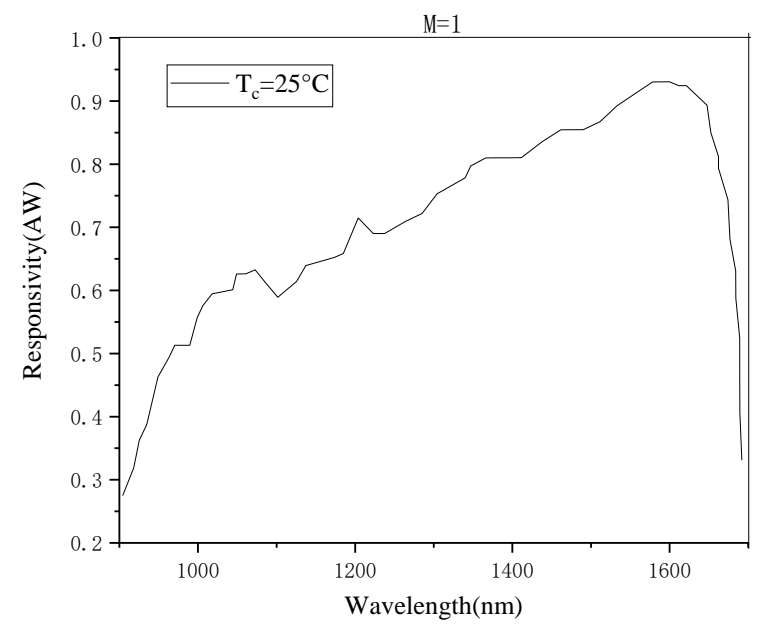

Figure 4: Spectral response curve

In Figure 4, when the wavelength is $1.57 \mu \mathrm{m}$, the conversion efficiency is the highest. In general, when the multiplication factor is larger, the conversion efficiency is also increased, and the performance of laser ranging can be improved [13]. The multiplication factor is the ratio of photocurrent $i_{s}$ generated by avalanche multiplication to photocurrent $i_{s 0}$ without avalanche effect. The multiplication factor is related to the reverse bias voltage applied on the PN junction and the material of the PN junction. The relationship between multiplication factor $\mathrm{M}$ and reverse bias voltage can be shown in equation (6):

$$
M=\frac{i_{s}}{i_{s 0}}=\frac{1}{1-\left(\frac{V}{V_{B}}\right)^{n}}
$$

In the equation, VB represents reverse bias, $\mathrm{V}$ represents applied reverse bias. $\mathrm{n}$ is usually taken as $1-3$, which depends on the properties of semiconductor materials, doping distribution and radiation wavelength. On this basis, if the multiplication factor is set too large, the shot noise will increase exponentially. The corresponding noise coefficient can be shown in equation (7):

$$
F_{m}=M\left[1-0.8\left(\frac{M-1}{M}\right)^{2}\right]
$$

It can be seen from the above equation that when the photoelectric signal is multiplied by $M$, the shot noise of the diode is multiplied by $M \sqrt{F_{m}}$ at the same time. The main function of photodetector is to convert the light signal power incident on the photosensitive surface of photodetector into corresponding current signal, as shown in equation (8):

$$
i(t)=\mathfrak{R} \cdot P(t)
$$

In the equation, $\mathrm{P}(\mathrm{t})$ represents the optical signal power and $\mathrm{i}(\mathrm{t})$ represents the corresponding current signal. $\mathfrak{R}$ is the voltage response of the detector, which can be expressed as equation (9):

$$
\mathfrak{R}=\frac{e \eta_{q}}{h v}
$$

In the equation, e is the electronic charge, $\eta_{\mathrm{q}}$ is the quantum efficiency of the detector, $h$ is the Planck constant, and $\mathrm{v}$ is the frequency of the incident light. The electric signal current is obtained by equation (10):

$$
I_{s}=\mathfrak{R} P_{r} M
$$

On this basis, the effective value of noise current can be approximately obtained by equation (11):

$$
I_{n}=\left[2 e\left(i_{s}+i_{b}+i_{d}\right) M^{2} F \Delta f+\frac{4 k_{B} T \Delta f}{R_{L}}\right]
$$

The signal-to-noise ratio equation of the detector output of the laser ranging system can be further obtained, as shown in (12):

$$
\begin{aligned}
S N R & =\frac{I_{S}}{I_{n}} \\
& =\frac{\mathfrak{R} P_{r} M}{\left[\begin{array}{l}
2 e\left(i_{s}+i_{b}+i_{d}\right) M^{2} F \Delta f \\
+\frac{4 k_{B} T \Delta f}{R_{L}}
\end{array}\right]}
\end{aligned}
$$

In the equation, $\mathrm{Pr}$ is the signal power received by the detector, $\mathrm{F}$ is the additional noise factor, $\Delta \mathrm{f}$ is the bandwidth of the receiving system, KB is the Boltzmann constant, and $\mathrm{T}$ is the absolute temperature [14].

In the process of remote detection, when measuring the distance, it can be obtained by equation (13):

$$
s=\frac{c t}{2}
$$

In the equation, $c$ is the speed of light and $t$ is the time of laser pulse flight. Therefore, the accuracy of ranging can be improved by accurately measuring the flight time of laser pulse. However, remote measurement also has a certain range, so it is necessary to find out the maximum operating distance first, as shown in equation (14):

$$
R=\left(\frac{2 P_{t} K_{t} K_{r} T_{\alpha}^{2} \rho A_{t} A_{r}}{\pi^{2} \theta^{2} P_{r \min }}\right)^{1 / 4}
$$

In the above equation, $\mathrm{P}_{\mathrm{t}}$ represents the laser power, $\theta$ represents the divergence angle, $K_{t}$ represents the efficiency of the emission system, and $\alpha$ represents the atmospheric attenuation coefficient. $A_{t}$ represents the target area, $A_{r}$ represents the lens 
area of the receiving system, and $\mathrm{K}_{\mathrm{r}}$ represents the transmittance of the receiving system. $\rho$ is the diffuse reflection coefficient of the target. $T_{\alpha}$ is the transmittance, that is, the degree of attenuation of the laser as it travels through the atmosphere. $\mathrm{T}_{\alpha}$ can be expressed by equation (15):

$$
T_{\alpha}=e^{-\alpha R}
$$

In the above equation, $\alpha$ represents the atmospheric attenuation coefficient.

\subsection{Error analysis}

In the process of ranging, the speed and flight time of light in the propagation will cause certain errors in ranging. The speed of light is less affected by the change of atmospheric refractive index, so the influence of this part can be ignored. The flight time is also affected by the pulse width of laser, the decay and distortion caused by atmospheric transmission, the influence of reflected target and optical receiving system on the pulse broadening and the accuracy of timing circuit. In general, in order to reduce drift error and time jitter, narrow pulse laser and appropriate pulse identification unit will be selected [15-16].

In the laser ranging system, the ranging accuracy depends on the total root mean square value of residual error and accidental error after system error correction. The ranging accuracy is shown in equation (16):

$$
S_{d}=\sqrt{\delta_{r}^{2}+\Delta R^{2}}
$$

In the equation, $\Delta \mathrm{R}$ represents the residual after system error correction, and $\delta_{\mathrm{R}}$ represents the total root mean square value of accidental error.

\subsection{Simulation test}

When receiving information, the noise is mainly composed of background light noise and transmission circuit noise. The noise emitted by the background light will interfere with the operation of the laser rangefinder [17]. Gaussian white noise is usually used for simulation, which can be expressed by equation (17):

$$
\rho\left(V_{n}\right)=\frac{1}{\sqrt{2 \pi} \sigma} e^{-\frac{\left(V_{n}-\mu\right)^{2}}{2 \sigma^{2}}}
$$

In the equation, $V_{n}$ represents the instantaneous noise voltage amplitude, $\mu$ is the mean value of noise, and $\sigma$ is the mean square difference of noise. Its random number is represented by equation (18):

$$
V_{n}=\frac{\sum_{i=1}^{m} u_{i}-m \mu}{\sqrt{m} \sigma}
$$

When $\mathrm{m}$ approaches $\infty, \mathrm{V}_{\mathrm{n}}$ is close to the ideal Gaussian distribution.

In the detection process, both DC signal and high frequency noise will cause certain impact.

Therefore, an ideal low-pass filter is needed to limit the bandwidth of signal and noise. Its impact response function is shown in equation (19):

$$
h(n)=\frac{w_{c}}{\pi} \sin c\left(w_{c} k \cdot \Delta t\right)
$$

In the equation, $\mathrm{w}_{\mathrm{c}}$ is the upper cut-off frequency of low-pass filter.

\section{Results and Discussion}

After the digital simulation of laser ranging, the Gauss white noise is obtained by Matlab software, as shown in Figure 5:

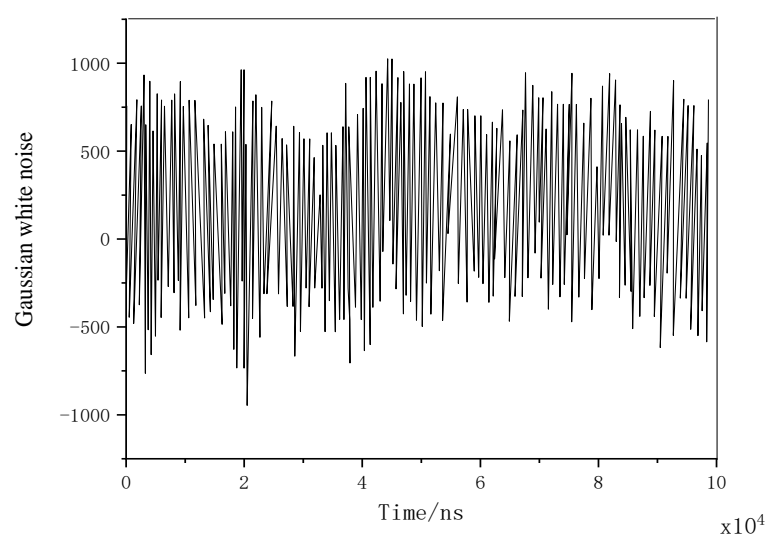

Figure 5: Gaussian white noise

On this basis, after superposition of laser echo, Figure 6 is obtained:

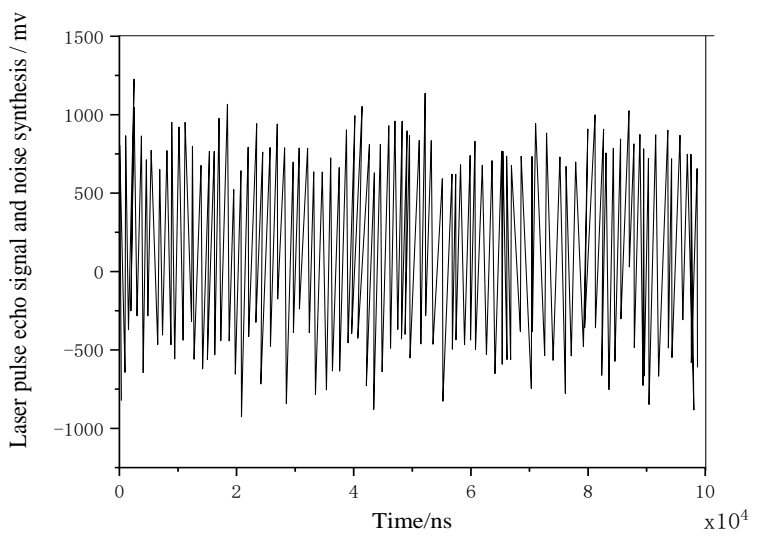

Figure 6: Superposition of laser echo and Gaussian white noise 
After filtering the superposition figure in Figure 6 with an ideal low-pass filter, the data in Figure 7 is obtained:

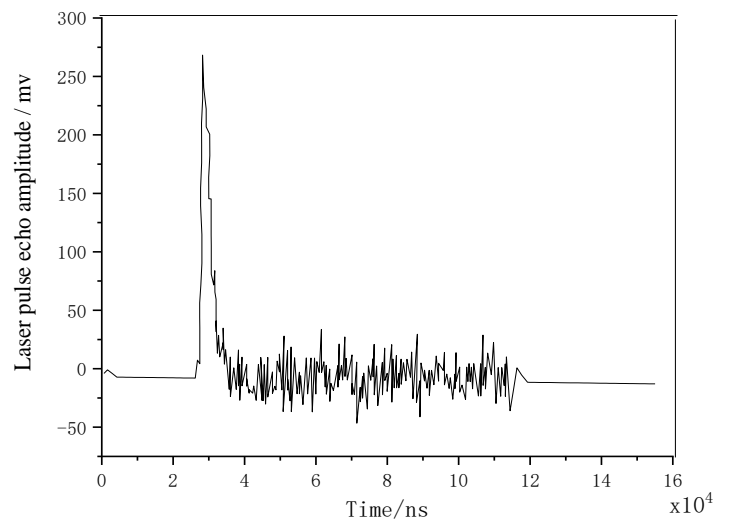

Figure 7: Laser echo filtered by ideal low pass filter

From the above Figure 5, Figure 6 and Figure 7, it can be seen that the filtered laser echo well describes the randomness of the noise, and each measurement result is different, which also indicates that there will be some errors in the ranging process. However, it can also be seen from the figure that although the results are different, their order of magnitude is fully consistent.

The output current of photomultiplier is $1.6 \times 10^{-}$ ${ }^{5} \mathrm{~A}$ and the signal-to-noise ratio is 19.1 . Therefore, the output voltage signal is as shown in Figure 8:

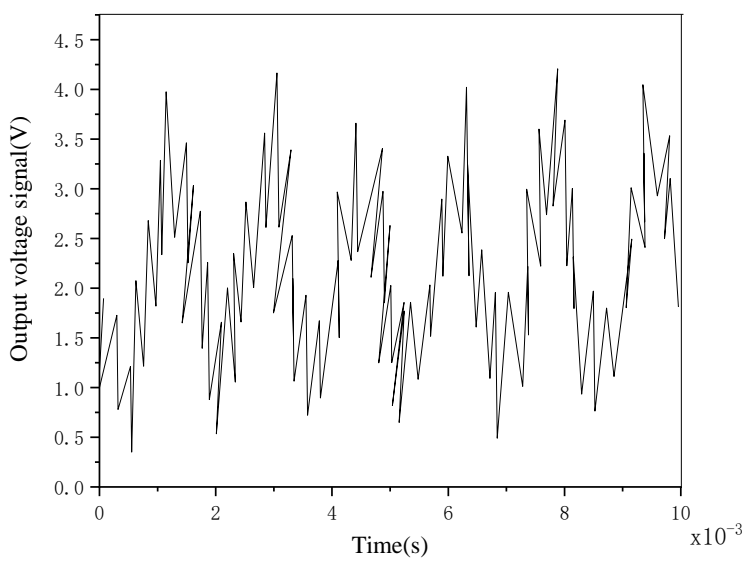

Figure 8: Output voltage signal

In Figure 8, the frequency is $20 \mathrm{kHz}$. It can be seen that the communication signal generator generates sinusoidal signal as the voltage source of LED and then generates sinusoidal light. Adjusting the signal frequency, the output signal of the system can be obtained.

It is also found that the error caused by laser pulse is $0.5 \mathrm{~m}$. In the laser receiving amplifier, the error caused by the bandwidth is $1.2 \mathrm{~m}$.

The error caused by the delay of the main echo is mainly due to the different circuit parameters of the main wave and the echo, and the error caused will not be changed due to other aspects.
Therefore, this part of error belongs to system error, which needs to be eliminated in the actual design. This error can be ignored when the variation of atmospheric pressure and temperature has little influence on the speed of light propagation and the range of distance measurement is small (less than $5 \mathrm{~km}$ ). Therefore, based on the above analysis, it can be obtained that the ranging error of the ranging system is $0.7 \mathrm{~m}$.

To sum up, in the process of robot manufacturing, in order to get a certain range of data when the robot is in use, photoelectric remote detection technology will be embedded in the manufacturing process to get more accurate data. Through the analysis of the photoelectric remote detection technology, starting with the laser ranging, ranging error and the influence factors of laser in the transmission process, it is found that when photoelectricity is transmitted in the atmosphere, the transmission characteristics of the atmosphere need to be considered, and the impact of the transmission characteristics should be reduced as much as possible in the practical application. The ranging errors are also in the range of deliberate acceptance. The simulation results also show that the technology can make some progress in detection.

\section{Conclusions}

In this study, through the analysis of the photoelectric remote detection imaging system, the photoelectric remote detection technology is further studied from the laser ranging, ranging error and the atmospheric transmission characteristics when photoelectricity is transmitted in the atmosphere, so that the technology can play a greater role in the robot manufacturing. In the research process, only the detection principle and ranging error in the photoelectric remote detection technology are studied, and the interference between different photoelectric signals is not analyzed, which will be further discussed in the later research. Therefore, the research on the photoelectric remote detection technology in robot manufacturing is of great significance for the development of photoelectric detection equipment and system, and provides a certain idea for improving the ranging level, and provides a theoretical basis for the experiment and application of the technology in other fields.

\section{References}

[1] Zhang X., Li H. (2018) Research on target capture probability calculation model of composite photoelectric detection imaging sensor system. Optik, 166. 161-168.

[2] Matsas E., Vosniakos G. C., Batras D. (2018) Prototyping proactive and adaptive techniques for human-robot collaboration in manufacturing 
using virtual reality. Robotics and ComputerIntegrated Manufacturing, 50. 168-180.

[3] Nielsen I., Dang Q. V., Bocewicz G, et al. (2017) A methodology for implementation of mobile robot in adaptive manufacturing environments. Journal of Intelligent Manufacturing, 28(5). 1171-1188.

[4] Hu L., Miao Y., Wu G, et al. (2019) iRobot-Factory: An intelligent robot factory based on cognitive manufacturing and edge computing. Future Generation Computer Systems, 90. 569-577.

[5] Urhal P., Weightman A., Diver C, et al. (2019) Robot assisted additive manufacturing: A review. Robotics and Computer-Integrated Manufacturing, 59. 335-345.

[6] Huang S., Shinya K., Bergström N, et al. (2018) Dynamic compensation robot with a new highspeed vision system for flexible manufacturing. The International Journal of Advanced Manufacturing Technology, 95(9-12). 4523-4533.

[7] Fan D., Bao C., Khan M. S, et al. (2018) A novel label-free photoelectrochemical sensor based on N, S-GQDs and CdS co-sensitized hierarchical $\mathrm{Zn2SnO4}$ cube for detection of cardiac troponin I. Biosensors and Bioelectronics, 106. 14-20.

[8] Pan A., Ma X., Huang S, et al. (2019) CsPbBr3 Perovskite Nanocrystal Grown on MXene Nanosheets for Enhanced Photoelectric Detection and Photocatalytic CO2 Reduction. The journal of physical chemistry letters, 10(21). 6590-6597.

[9] Kim H. S., Kumar M. D., Kim J, et al. (2018) Vertical growth of MoS2 layers by sputtering method for efficient photoelectric application. Sensors and Actuators A: Physical, 269. 355-362.

[10] Bhola R., Krishna N. H., Ramesh K. N, et al. (2018) Detection of the power lines in UAV remote sensed images using spectral-spatial methods. Journal of environmental management, 206: 1233-1242.

[11] Liu Q., Liu Z., Xu W, et al. (2019) Human-robot collaboration in disassembly for sustainable manufacturing. International Journal of Production Research, 1-18.

[12] Bai X., Wang W., Sun Y, et al. (2018) Operating performances of an ASHP unit operated in a mild and humid region using tube-encircled photoelectric sensor based defrosting initiation strategy. Energy and Buildings, 177. 140-153.

[13] Sadrfaridpour B., Wang Y. (2017) Collaborative assembly in hybrid manufacturing cells: An integrated framework for human-robot interaction. IEEE Transactions on Automation Science and Engineering, 15(3). 1178-1192.

[14] Xin J., Kaixuan Z., Jiangtao J, et al. (2018) Design and implementation of Intelligent transplanting system based on photoelectric sensor and PLC. Future Generation Computer Systems, 88. 127139.

[15] Paik J. (2018) Soft robot design methodology for 'push-button'manufacturing. Nature Reviews Materials, 3(6). 81.

[16] Urhal P., Weightman A., Diver C, et al. (2019) Robot assisted additive manufacturing: A review. Robotics and Computer-Integrated Manufacturing, 59. 335-345.

[17] Wang Z., Chang T., Zeng X, et al. (2019) Fiber optic multipoint remote methane sensing system based on pseudo differential detection. Optics and Lasers in Engineering, 114. 50-59.

[18] Yang X., Sun H., Fu K, et al. (2018) Automatic ship detection in remote sensing images from google earth of complex scenes based on multiscale rotation dense feature pyramid networks. Remote Sensing, 10(1). 132. 